\title{
Mercurio en tiburón Sphyrna lewini y Carcharhinus falciformis del pacífico guatemalteco
}

\author{
Eduardo E. Chacón, Adrián M. Castro, Alva J. Montiel, Juan M. Tejeda, Gustavo A. Elías* \\ Centro de Estudios del Mar y Acuicultura, Universidad de San Carlos de Guatemala, Guatemala.
}

*Autor al que se dirige la correspondencia: tavoelias@hotmail.com

Recibido: 18 de septiembre 2015 / Revisión: 29 de marzo 2016 / Aceptado: 16 de septiembre 2016 / Disponible en línea: 13 de marzo 2017

\section{Resumen}

$\mathrm{E}$ 1 objetivo del estudio fue determinar la presencia de mercurio en muestras de carne de dos especies de tiburón: Sphyrna lewini y Carcharhinus falciformis, acopiados por la pesca artesanal en dos puntos de la costa pacífica de Guatemala. Con el análisis de la información se verificó el estado de inocuidad de los organismos respecto a las concentraciones de mercurio, utilizando como base la normativa europea. Se recolectaron 66 muestras, durante 10 meses, las cuales fueron analizadas utilizando la técnica de espectrofotometría de absorción atómica. Posteriormente se realizó un análisis descriptivo y se aplicó la ecuación del Índice de Peligro Sistémico (IP). El análisis de resultados indicó que 16 (73\%) organismos de S. lewini, presentaron concentraciones de mercurio superiores al límite máximo permisible (LMP) de acuerdo a la normativa europea ( $1 \mathrm{mg} / \mathrm{kg}$ de peso fresco), aunque el IP $\left(1.626857143 \times 10^{-4}\right)$ demostró que no existe riesgo potencial para la salud de los consumidores, además que esta especie no muestra correlación significativa $(\rho=0.292)$, entre el tamaño del organismos y la concentración de mercurio. Únicamente 4 (9\%) organismos de la especie $C$. falciformis, presentaron concentraciones de mercurio arriba del límite máximo permisible de acuerdo a la normativa europea, y el cálculo de IP $\left(4.345714286 \times 10^{-5}\right)$, indicó que el músculo de dicha especie puede consumirse sin ningún riesgo potencial para las personas.

Palabras claves: contaminación, bioacumulación, pesca, límite máximo permisible, metil-mercurio.

\section{Abstract}

$\mathrm{T}$ he objective of the study was to determine the presence of mercury in samples of meat corresponding to two shark species: Sphyrna lewini and Carcharhinus falciformis collected by artisanal fisheries in two points of the Pacific coast of Guatemala. With the analysis of information safety status of sharks for concentrations of mercury was verified, using as a basis the European regulations. Sixty six samples were collected in 10 months, these samples were analyzed using the atomic absorption spectrophotometry technique. After a descriptive analysis and the equation of Systemic Risk Index (RI) was applied. The analysis results indicated that 16 (73\%) organisms of S. lewini, had concentrations above the permissible limit according to European standards ( $1 \mathrm{mg} / \mathrm{kg}$ wet weight) mercury, although the rate of RI $\left(1.626857143 \times 10^{-4}\right)$ it proved there is not a potential health risk to consumers, besides this species shows no significant correlation $(\rho=0.292)$, between the size of organisms and the concentration of mercury. Only four organisms (9\%) of the species $C$. falciformis presented mercury concentrations above the permissible limit according to European standards, and the calculated RI (4.345714286 x $\left.10^{-5}\right)$ showed that the muscle of theses species can be eaten without any risk to consumers.

Key words: shark, mercury, bio-accumulation, fishing, permissible limit allowable, metilmercury. 


\section{Introducción}

Los océanos presentan serios problemas de impacto ambiental, que tienen su origen en diversas fuentes. Los tiburones son depredadores ubicados en el extremo de las cadenas tróficas, tanto costeras como oceánicas, por lo tanto sus tejidos permiten advertir niveles tóxicos de metales pesados (Galván, Polo, Barrera, Escobar, \& Torres, 2014).

La fuente más importante de mercurio en la dieta humana son los peces, el $80 \%$ de este elemento se encuentra en forma orgánica, principalmente metilmercurio (Hurtado, 2010). Doadrio (2004) indica que en ciertas poblaciones que basan su alimentación en el consumo de peces y otros productos obtenidos del mar, pueden llegar a sobrepasar la ingesta máxima tolerable por semana de mercurio, fijada en $300 \mu \mathrm{g}$ de mercurio total por persona, y en $200 \mu \mathrm{g}$ cuando se trata de metilmercurio. Casi el 100\% del mercurio que se bioacumula en peces depredadores como los tiburones, es metilmercurio, se han obtenidos concentraciones de hasta $3 \mathrm{mg} / \mathrm{kg}$ (Velasco-González, Echavarría-Almeida, Pérez-López, \& Villanueva-Fierro, 2001).

El metilmercurio es una de las formas químicas que se encuentran mayormente en alimentos, principalmente en productos pesqueros. El metilmercurio es altamente tóxico, ya que se acumula en el tejido nervioso de los organismos, convirtiéndose en un problema de salud pública. La población que consume pescado contaminado, es la que está mayormente expuesta (León $\&$ Peñuela, 2011).

El contenido máximo de mercurio en productos de la pesca según normativa europea, se establece en $0.5 \mathrm{mg} / \mathrm{kg}$ de peso fresco, salvo para determinadas especies, como el bonito y tiburones que se permite $1 \mathrm{mg} /$ kg de peso fresco (World Health Organization, 1991).

Holmes, James y Levy (2009), reportan que todas las clases de peces incluyendo las especies exóticas, entre ellas los tiburones presentan niveles de mercurio arriba de $1.52 \mathrm{mg} / \mathrm{kg}$. Se consideró como objeto de estudio a los tiburones ya que son organismos de gran aceptación comercial dentro de la dieta alimenticia de los guatemaltecos debido a su disponibilidad, bajo precio y buen sabor, son especies sujetas a explotación pesquera por parte de la pesca artesanal en todo el litoral Pacífico de la república. Los tiburones están en la cúspide de las cadenas alimenticias de los ecosistemas oceánicos (Kiszka et al., 2015), siendo susceptibles a la bioacumulación.
La investigación tuvo como propósitos: determinar los niveles de mercurio presentes en el músculo de tiburón Sphyrna lewini y Carcharhinus falciformis, establecer la correlación entre el tamaño de captura de los organismos y la concentración de mercurio y, por último, determinar el índice de peligro sistémico (IP) de las muestras obtenidas. Las especies $C$. falciformis y $S$. lewini, fueron consideradas en la investigación, ya que Ruiz y López (1999), las consideran como especies de tiburón de importancia comercial en Guatemala.

\section{Materiales y métodos}

\section{Muestreo}

Para el estudio se utilizó un muestreo por selección intencionada, colectando en los centros de acopio de acuerdo a su disponibilidad cada mes, ejemplares de tiburón S. lewini y C. falciformis fueron colectados directamente de los centros de acopio de los pescadores artesanales de Puerto de San José y Puerto de Iztapa, Escuintla, en el Pacífico de Guatemala.

En un período de 10 meses se recolectaron 66 muestras de músculo de tiburón, 22 correspondieron a la especie $S$. lewini, y 44 a la especie $C$. falciformis. La extracción del músculo se realizó in situ en los sitios de acopio de las capturas.

Se colectaron muestras con un peso de $454 \mathrm{~g}$ y se colocaron en bolsas plásticas herméticas selladas y rotuladas. Posteriormente fueron trasladadas al laboratorio y sometidas a refrigeración para su adecuada conservación. Para cada muestra se llenó una ficha de control, en la cual se indicó el peso total del espécimen, sitio de procedencia, especie y fecha de captura.

\section{Análisis de laboratorio}

El análisis de las muestras se realizó en la empresa de Investigación, Laboratorio, Análisis, Servicios y Asesoría (Inlasa), acreditada ISO 17025: 2005, OGALE- 008-05. El procedimiento para dicho análisis fue el siguiente:

La muestra se digería con una solución de ácido sulfúrico para liberar todos los compuestos de mercurio orgánico, el cual pasa a mercurio iónico. Una reducción adicional del mercurio iónico a mercurio metálico utilizando cloruro estañoso, hace posible la medición del mercurio gaseoso en el aire. El límite de detección de este método es de $0.01 \mu \mathrm{g}$ de mercurio, para poder 
leer por espectrometría de absorción atómica con celda de vapor frío. (FIAS- Flow Injection Analysis System, Sistema Analítico de Flujo de Inyección) utilizando un aparato AA Shimadzu 6800.

\section{Definición de las variables}

La variable dependiente considerada en la investigación fue la concentración de mercurio presente en el músculo de los organismos, siendo el indicador la concentración de $\mathrm{Hg}$ en $\mathrm{mg} / \mathrm{kg}$ de muestra.

\section{Análisis de la información}

Se hizo un análisis descriptivo calculando las medias y varianza de los valores de concentración de mercurio en cada una de las muestras. Posteriormente se calculó el número y porcentaje de muestras de ambas especies que sobrepasaron el límite máximo permisible (LMP), tomando como referencia la normativa europea de $1 \mathrm{mg} / \mathrm{kg}$ de peso fresco.

Los resultados se agruparon de acuerdo a las variables dependientes. Se utilizó el coeficiente de Spearman para realizar correlaciones entre el tamaño de captura del tiburón, especie y concentración de mercurio. Además, se calculó el IP de la variable dependiente, para cada una de las especies estudiadas.

$\mathrm{IP}=$ Dosis de exposición/Dosis de referencia.

Dosis de exposición $=(\mathrm{C})(\mathrm{TI})(\mathrm{TA}) / \mathrm{PC}$

$\mathrm{C}=$ Concentración promedio del metal $(\mathrm{mg} / \mathrm{kg})$;

$\mathrm{TI}=$ Tasa de ingestión diaria de pescado $(0.0065 \mathrm{~kg} /$ día);

$\mathrm{TA}=$ Tasa de absorción del metal por el tracto gastrointestinal $(10 \%)$.

$\mathrm{PC}=$ Peso corporal $70 \mathrm{~kg}$ (United States Environmental Protection Agency, 1989)

\section{Resultados}

S. lewini mostró la mayor media de concentración de mercurio, y también presentó mayor dispersión en las concentraciones detectadas. La especie $C$. falciformis presentó una media inferior al LMP $(1 \mathrm{mg} / \mathrm{kg})$ y sus concentraciones no fueron tan dispersas en los diferentes organismos (Tabla 1).

S. lewini presentó mayores concentraciones de mercurio arriba del LMP en comparación con la especie C. falciformis que mostró un bajo nivel de contaminación (Tabla 2).

Tabla 1

Promedio y varianza de las concentraciones de mercurio, para cada una de las dos especies muestreadas

\begin{tabular}{llll}
\hline Especies & $\mathrm{N}$ & $\mathrm{X}$ & $\sigma$ \\
\hline Sphyrna lewini & 22 & 1.94471 & 1.7958 \\
Carcharhinus falciformis & 44 & 0.527541 & 1.0702 \\
\hline
\end{tabular}

Nota. Concentraciones en mg/kg. Metodología: mercurio PC-FQ-018

Tabla 2

Contenido de mercurio en muestras de C. falciformis $y \mathrm{~S}$. lewini

\begin{tabular}{lcc}
\hline Resultado & \multicolumn{2}{c}{ Valores } \\
\cline { 2 - 3 } & C. falciformis & S. lewini \\
\hline Número de muestras extraídas & 44 & 22 \\
Número de muestras con valores detectables & 44 & 22 \\
Muestras con valor detectable (\%) & 100 & 100 \\
Número de muestras con valores por encima de LMP de la normativa europea (1 mg/Kg) & 4 & 16 \\
Muestras con valores por encima del LMP (\%). & 9 & 73 \\
\hline
\end{tabular}

Nota. Límite detección $25 \mu \mathrm{g} / \mathrm{kg}$ 
Los datos recabados durante la investigación revelan que no existe correlación entre la talla de los organismos y la concentración de mercurio (Figura 1), pero se observa que la variabilidad de la concentración de mercurio en $S$. lewini, es más alta que $C$. falsiformis.

La especie $S$. lewini presentó una tendencia por arriba del LMP (84\%) de los organismos muestreados durante la época de captura. Mientras que la especie $C$. falciformis muestra una tendencia a presentar valores de concentración de mercurio debajo de los LMP en la temporada a.

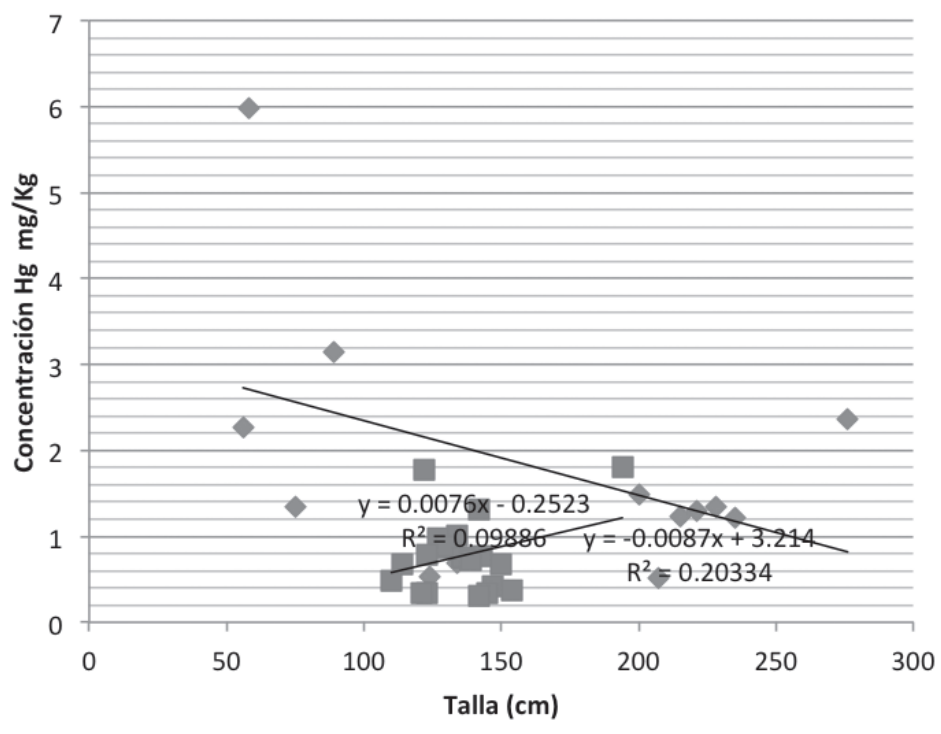

- Concentración Hg. Sphyrna lewini

Concentración Hg. Cacharhinus falciformis

__Lineal (Concentración Hg. Sphyrna lewini)

—Lineal (Concentración Hg. Cacharhinus falciformis)

Figura 1. Distribución de la concentración de mercurio presente en $S$. lewini y $C$. falciformis en función de la talla (longitud) de los organismos muestreados. C. falciformis $\mathrm{n}=20, S$. lewini $\mathrm{n}=13$.

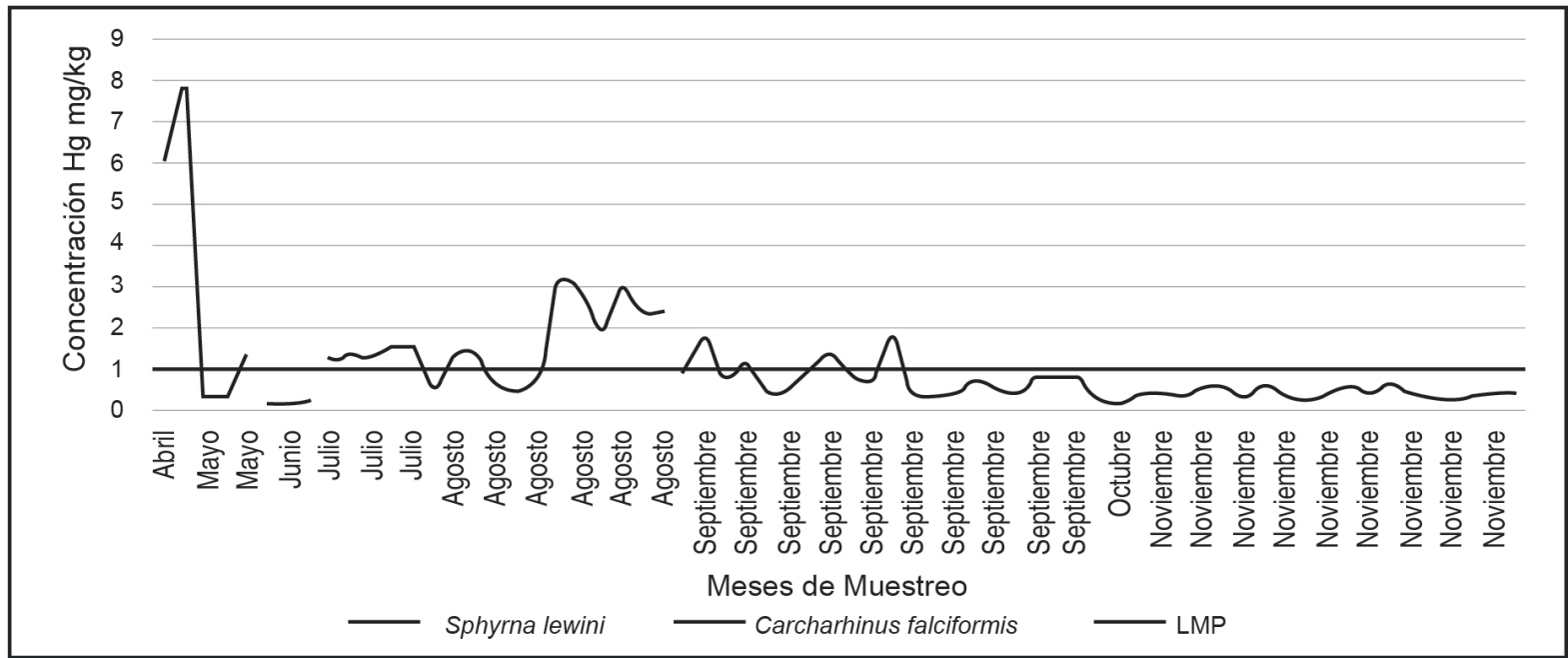

Figura 2. Comportamiento de la concentración de mercurio en los meses de muestreo. La especie $S$. lewini, tiene un comportamiento estacional, $C$. falciformis no se muestreó en los meses de mayo-agosto. 


\section{Discusión}

De las 22 muestras analizadas, para la especie $S$. lewini, 16 (73\%) presentaron concentraciones de mercurio arriba del LMP (1 mg/kg) (Tabla 2), con valores de $1.94471 \pm 1.7958 \mathrm{mg} / \mathrm{kg}$, pero al determinar el IP $\left(1.62685714 \times 10^{-4}\right)$, se puede establecer que no existe un riesgo a la salud de los consumidores. Estudios realizados por Hurtado (2010), establecieron concentraciones con valores de $0.82 \pm 0.33 \mathrm{mg} / \mathrm{kg}$ de mercurio en juveniles de $S$. lewini. En otras especies cercanas a los tiburones como los batoideos, se reportan concentraciones de mercurio mucho más bajas en rangos que varían de 0.02 a $0.61 \mathrm{mg} / \mathrm{kg}$ de peso (Farrugia, Oliveira, Knue, \& Seitz, 2015), así mismo S. lewini presenta las mayores concentraciones de monometilmercurio, tanto en aletas secas, como en sopa de aletas de tiburón, (Nalluri et al., 2014), lo cual reafirma que S. lewini es una especie del Pacífico de Guatemala con altos niveles de mercurio. Es importante considerar aquí que esta especie es fisoria y consume más invertebrados, en comparación con C. falsiformis que es una especie epilelágica que consume principalmente peces.

Con relación a la especie $C$. falciformis se puede observar en la Tabla 2, que de 40 sub-muestras, el $91 \%$ no sobrepasa el LMP, con valores de $0.527541 \pm 01.0702$ $\mathrm{mg} / \mathrm{kg}$, muestra que está especie está menos contaminada de mercurio, siendo un buen indicador, ya que fue la especie que más se capturó durante el tiempo de la investigación y como consecuencia la que más se consume en el país. El IP $\left(4.345714286 \times 10^{-5}\right)$, para la especie también establece que no existe ningún riesgo de contaminación por mercurio para la salud de los consumidores potenciales, lo cual coincide con Escribá, Soto y García (2007), quienes reportan que los niveles de mercurio encontrados en muestras de carne de tiburón de las costas de Guatemala, son menores a las concentraciones presentadas por la FAO/WHO-EPA. De las concentraciones de mercurio encontradas, las de C. falciformis son más bajas que las reportadas en tiburones azules Prionace glauca del sur oeste del Océano Índico, que reportan cantidades de mercurio de $5.3 \pm 2.17 \mathrm{mg} / \mathrm{kg}$ y en Baja California $1.96 \pm 1.48 \mathrm{mg} / \mathrm{kg}$ (Maz-Courrau et al., 2012).

Al observar las Figuras 1 y 2 , se puede concluir que existe una diferencia en la frecuencia de concentración de mercurio en la carne de las dos especies, siendo significativamente mayor en $S$. lewini.

El patrón de distribución de la frecuencia de aparecimiento de muestras contaminadas de mercurio en
S. lewini, en el tiempo muestra una tendencia irregular, mientras que la especie $C$. falciformis presenta poca variabilidad en función del tiempo de captura y el $91 \%$ de los datos, están debajo de LMP (Figura 2).

Los datos recabados de talla de los organismos de las dos especies estudiadas versus concentraciones de mercurio, se sometieron a la prueba de Spearman $(\alpha 0.05)$, indicando que no existe correlación entre las dos variables, lo cual se puede confirmar en la Figura 1.

La falta de correlación entre el tamaño de los organismos y la concentración de mercurio, es ratificada en investigaciones realizadas por Sánchez (2010).

\section{Agradecimientos}

Este proyecto de investigación se llevó a cabo gracias al financiamiento proporcionado por la Dirección General de Investigación (Digi) de la Universidad de San Carlos de Guatemala (Puirna No. 4.8.26.2.44). Agradecemos a los pescadores artesanales que nos apoyaron con la recolección de muestras y nos brindaron información que fue útil durante la ejecución del proyecto.

\section{Referencias}

Doadrio, A. L. (2004). Ecotoxicología y Acción toxicología de mercurio. Anales de la Real Academia de Farmacia, 70, 933-959.

Escribá, C., Soto, M., \& García, J. (2007). Cuantificación de mercurio $(\mathrm{Hg})$, por la técnica de espectrometría de absorción atómica con celda de vapor frío (FIAS), en muestras de carne de tiburón provenientes de las Costas del Atlántico y Pacífico de la República de Guatemala (Tesis de licenciatura). Universidad de San Carlos de Guatemala, Facultad de Ciencias Química y Farmacia, Guatemala. Recuperado de http://biblioteca.usac.edu. gt/tesis/06/06_2503.pdf.

Farrugia, T., Oliveira, A., Knue, J., \& Seitz, (2015). Nutritional content mercury and trace element analysis of two skate (Rajidae) species in the Gulf of Alaska. Journal of Food Composition and Analysis, 42, 152-163. doi: 10.1016/j. jfca.2015.03.013

Galván, F., Polo, C. J., Barrera, A. M., Escobar, O., \& Torres, Y. E. (2014). Los Tiburones como bioindicador de contaminantes y cambios tróficos en 
los ecosistemas marinos. En C. A. González, A. Vallarino, J. C. Pérez \& A. M. Low (Eds.), Bioindicadores: Guardianes de nuestro futuro ambiental (pp. 375-396). México D. F.: El colegio de la frontera Sur e Instituto Nacional de Ecología y Cambio Climático.

Holmes, P., James, K. A., \& Levy, L. S. (2009). Is low-level environmental mercury exposure of concern to human health? Science of the Total Environmental, 408(2), 171-182. doi:10.1016/j. scitotenv.2009.09.043

Hurtado, R. (2010). Determinación de mercurio total en hígado y músculo de tiburones provenientes de las pesquerías de Sonora y Sinaloa, México (Tesis de maestría). Universidad de Sonora, México. Recuperada de http://www.posgradoenbiociencias. uson.mx/produccion/docs/Tesis_Rocio_Hurtado. pdf

Kiszka, J. J., Aubail, A., Hussey, N. E., Heithanus, M. R., Caurant, F., \& Bustamante, P. (2015). Plasticity of trophic interactions among sharks from the oceanic south-western Indian Ocean revealed by stable isotope and mercury analyses. Deep Sea Research Part I. Oceanographic Research Papers, 96, 49-58. doi:10.1016/j.dsr.2014.11.006

León, D. E., \& Peñuela, G. A. (2011). Trascendencia del metilmercurio, en el ambiente, la alimentación y la salud humana. Producción + Limpia, 6(2), 108-116.

Maz-Courrau, A., López-Vera, C., Galván-Magaña, F., Escobar-Sánchez, O., Rosíles-Martínez, R., \& Sanjuán-Muñoz, A. (2012). Bioaccumulation and biomagnification of total mercury in four exploited shark species in the Baja California península, México. Bulletin of Environmental Contamination and Toxicology, 88(2), 129-134. doi:10.1007/s00128-011-0499-1

Nalluri, D., Baumann, Z., Albercrombie, D. L., Chapman, D. D., Hammerschmidt, C. R., \& Fisher, N. S. (2014). Methylmercury in dried shark fins and shark fin soup from American restaurants. Science of the Total Environment, 496, 644-648. doi:10.1016/j.scitotenv.2014.04.107

Ruiz, C. L., \& López, N. M. (1999). Estudio sobre la pesquería de tiburón en Guatemala. Organización de las Naciones Unidas para la Alimentación y la
Agricultura. Recuperado de http://www.fao.org/ docrep/003/x2097e/x2097E08.htm

Sánchez, O. (2010). Bioacumulación y biomagnificación de mercurio y selenio en peces pelágicos, mayores de la costa occidental de Baja California Sur (Tesis de doctorado). Instituto Politécnico Nacional, México. Recuperada de www.repositoriodigital.ipn.mx

United States Enviromental Protection Agency. (1989). Risk Assessment Guidance for superfund Volume I. Human Health. Evaluation Manual (Part A) EPA/540/1-89/002. Washington, D.C.: Office of Emergency and Remedial Response.

Velasco-González, O., Echavarría-Almeida, S., Pérez-López, M., \& Villanueva-Fierro, I. (2001). Contenido de Mercurio y Arsénico en atún y sardinas enlatadas mexicanas. Revista Internacional de Contaminación Ambiental, 17(1), 31-35.

World Health Organization \& International Programme on Chemical Safety. (1991). Inorganic mercury. En L. Friberg, Environmental Health Criteria 118. Geneva: Autor. Recuperado de http://apps. who.int/iris/bitstream/10665/40626/1/IPCS EHC_118.pdf 